\title{
Current diagnosis and treatment of rhinosinusal aspergilloma (Review)
}

\author{
DANIELA VRINCEANU ${ }^{1 *}$, MIHAI DUMITRU ${ }^{1 *}$, OANA MARIA PATRASCU $^{2}$, \\ ADRIAN COSTACHE ${ }^{2}$, TOMA PAPACOCEA ${ }^{3 *}$ and ROMICACERGAN ${ }^{4}$ \\ ${ }^{1}$ ENT Department, Bucharest Emergency University Hospital, 010271 Bucharest; \\ ${ }^{2}$ Department of Pathology, 'Carol Davila' University of Medicine and Pharmacy, 020021 Bucharest; \\ ${ }^{3}$ Department of Neurosurgery, 'Sf. Pantelimon' Hospital, 021661 Bucharest; ${ }^{4}$ Department of Anatomy, \\ 'Carol Davila' University of Medicine and Pharmacy, 050474 Bucharest, Romania
}

Received June 2, 2021; Accepted July 2, 2021

DOI: 10.3892/etm.2021.10699

\begin{abstract}
There are numerous types of sinusitis caused by fungal strains, some of which already colonize the nasal cavity. Mild forms present fungus balls growing inside a preexisting sinus cavity. The invasive type ranges from chronic manifestations to acute aggravated episodes. The latter scenario is encountered in cases with reduced immune responses, such as patients with diabetes, individuals receiving any form of transplant, AIDS cases and chemotherapy patients. Without the control of immunosuppression, the infection is aggravated and extends to the orbit and inside the skull base, regardless of the prompt surgical and medical treatment. This is the most common pathogenic fungus on the nasal sinuses level. It can occasionally enter the sinus cavity during dental procedures. The pathogenesis is enhanced by anaerobic conditions in poorly ventilated sinus cavities. Rhinosinusal aspergilloma has a slow, insidious evolution over months and even years. Our experience revealed the presence of both a dental problem and previous self-administered antibiotic regimens in almost every case. The initial symptoms are common with sinusitis of dental origin, but aspergilloma should be considered when a patient with a competent immune system does not respond to standard antibiotic treatment. The final diagnosis of rhinosinusal aspergilloma is conducted on a pathology sample with silver staining. The bacteriology exam of the sinus secretion rarely reveals a fungus infection; however, as revealed in our clinical experience, there may be coinfection with other multidrug-resistant bacteria. Surgical treatment must establish a wide exposure of the sinus cavity and correct drainage
\end{abstract}

Correspondence to: Dr Adrian Costache, Department of Pathology, 'Carol Davila' University of Medicine and Pharmacy, 37 Dionisie Lupu Street, 020021 Bucharest, Romania

E-mail: adriancostacheeco@yahoo.com

*Contributed equally

Key words: rhinosinusal, aspergilloma, diagnosis, treatment, pathology regardless of the external, combined or endoscopic approach. Early diagnosis and emergency surgical debridement along with administering systemic antifungal compounds in some cases represent the key to the successful treatment of invasive aspergilloma.

\section{Contents}

1. Introduction to fungal rhinosinusitis

2. General data on rhinosinusal aspergilloma

3. Principles of diagnosis in rhinosinusal aspergilloma

4. Principles of treatment in rhinosinusal aspergilloma

5. Conclusions

\section{Introduction to fungal rhinosinusitis}

PubMed was searched using the key words 'sinus and aspergilloma' and limited the search to free full text available articles. All 20 articles identified were included in this review and other specific aspects were clarified also using free full text sources on PubMed or established reference ENT books. Moreover, concerning the time frame, all references were not older than 1973. Applying other inclusion criteria would have limited the scope of this review.

There are numerous types of sinusitis caused by fungal strains, some of which already colonize the nasal cavity. Mild forms present fungus balls growing inside a preexisting sinus cavity. The invasive type ranges from chronic manifestations to acute aggravated episodes. The latter scenario is encountered in cases with reduced immune responses, such as patients with diabetes, individuals receiving any form of transplant, AIDS cases and chemotherapy patients. Without the control of immunosuppression, the infection is aggravated and extends to the orbit and inside the skull base, regardless of the prompt surgical and medical treatment. Conversely, the cases with chronic invasive sinusitis in individuals with a competent immune response present a slow evolution with minimum vascular invasion $(1,2)$.

Infectious organisms involved in fungal aggressive sinusitis are most frequently members of the Aspergillus family, Mucor, 
Alternaria, Auricularia, Bipolaris, Candida, Drechslera, Sporothrix Schenckii and Pseudallescheria Boydii. One of the first classification systems is based on the histology aspect. Therefore, the cases with fungal rhinosinusitis lacking a granulomatous response are associated with a reserved prognosis due to a high rate of persistence and recurrence. However, these data are not available during the clinical exam and should not influence the first line of treatment. The pathology result is key to a final diagnosis and granuloma-related prognosis in fungal rhinosinusitis $(3,4)$.

Noninvasive forms of fungal rhinosinusitis were recently defined as allergic fungal rhinosinusitis (AFRS). Although there are still debates regarding its pathogenesis, this appears to be the most common type.

For defining AFRS, the following criteria must be used: type I hypersensitivity, nasal polyposis, specific computed tomography (CT) scan aspect of a dense sinus cavity content (Fig. 1), positive fungal culture, or a positive pathology slide stained accordingly and lack of tissue invasion. Supplementary data are elevated total IgE serum values, the presence of specific IgE antigens and peripheral blood eosinophilia $(5,6)$.

AFRS is mostly unilateral and may produce bone erosions with orbit and skull base invasion in advanced stages. When surgically opening the fungal cavity, the content is greenish and with high consistency. From a pathological point of view, this secretion contains eosinophils, Charcot-Leyden crystals, and fungal hyphae. The fungal hyphae are thin, uniform and regularly septate, with dichotomous branching at 45 degrees. The host response is minimal, with no inflammatory aspects of the tissue and no fungal invasion, although cases of invasive and extensive aspergillosis have been reported (Fig. 2) (7). The detail differentiating AFRS from chronic eosinophilia rhinosinusitis is the presence of a fungal allergy. AFRS is not related to the presence of fungi in the cavity but more to the aberrant response to environmental naturally occurring fungus. Using laboratory procedures with high sensitivity, fungi colonizing the nasal cavity may be detected in almost any individual. Given the wide range of fungi involved in developing AFRS, it is necessary to identify the precise type to prepare patient desensitization. Another aspect is that patients rarely present associated dermal mycosis (8).

\section{General data on rhinosinusal aspergilloma}

Aspergillus is a fungus present worldwide, and a member of the ascomycota class. Along with Penicillium, they form the Aspergillaceae family. This fungus usually evolves in moist areas and agricultural environments or on the surface of decomposing organic residues. Its growth is through the process of budding or branching. It can be easily carried by wind and inhaled. The branching microorganisms are easily identified with silver stains. This is the most common pathogenic fungus at the level of the nasal sinuses. Occasionally, it can enter the sinus cavity during dental procedures. The pathogenesis is enhanced by anaerobic conditions in poorly ventilated sinus cavities $(9,10)$.

After the intake of spores of Aspergillus fumigates, Aspergillus flavus, or Aspergillus niger an infection is generated, usually localized in the lungs of immunocompromised patients. However, destructive lesions occasionally appear in the paranasal sinuses, anterior palate, or nasal cavities. There have been cases of aspergilloma recorded even in immunocompetent hosts (Fig. 3). Chronic manifestations occur in healthy subjects, while acute manifestations burden immunocompromised hosts. Invasive forms in healthy subjects are extremely rare. Since the first description in 1953 as an opportunistic infection, numerous cases have been frequently documented during the time of an autopsy $(11,12)$.

Aspergilloma defines infections in the upper airways or lungs, cutaneous manifestations, or extrapulmonary sites, along with allergic reactions produced by the Aspergillus species. The most frequent site of infection are the lungs, with a maximum of $20 \%$ of cases also presenting with sinus infections according to some studies. Fungal sinusitis accounts for $\sim 6 \%$ of the cases with sinusitis. The most common pathogen is Aspergillus fumigatus followed by Aspergillus flavus and Aspergillus niger. The most common site of infection is the maxillary sinus $(13,14)$.

Rhinosinusal aspergilloma is also classified into invasive and noninvasive, depending on the mucosa involvement and bony erosion. Invasive aspergilloma may evolve into a malignant state, with the destruction of the adjacent orbit or skull base $(15,16)$.

Differential diagnosis includes Wegener granulomatosis and Pseudomonas lesions. Pathology reveals the progression of the infection in deep tissues. Moreover, there is a vascular invasion with tissue necrosis (Fig. 4). The prognosis of such cases with a poor immune response is uncertain $(17,18)$.

\section{Principles of diagnosis in rhinosinusal aspergilloma}

Common complaints are nasal obstruction, rhinorrhea, and smell dysfunction. The clinical exam should underline the fact that the symptoms appear only on one side of the nasal cavity. Facing such a scenario, the possibility of sinusitis with dental origin or fungal sinusitis associated with nasal polyposis should be considered. Occasionally, these two scenarios may intertwine (19). Unilateral, persistent headache, trigeminal neuralgia in the V2 territory, posterior rhinorrhea and unilateral cacosmia are clinically strong arguments for a possible odontogenic rhinosinusitis associated with aspergilloma.

Chronic evolution of a sinus infection due to a dental problem in an immunocompetent patient may lead to aspergilloma through a range of mechanisms: repeated use of antibiotics, closed sinus cavity due to ostium obstruction, exhaustion of the immune resources of the host due to chronic bacterial infection (20).

Clinical examination of the ENT with nasal endoscopy may reveal the presence of a purulent secretion in the middle meatus that drains on the tail of the lower cornet, marked edema or polyps in the middle meatus. After anemia and aspiration, it is possible to highlight aspergilloma even in the middle meatus, in the nasal fossa or in the sphenoethmoidal recess (Fig. 5). It is important to gently clean the nostrils before the examination, in order to avoid bleeding and highlight the lesions. Careful examination of the teeth on the homonymous hemimaxillary that can identify the presence of a mobile, painful tooth or an old dental work under which the source of origin for odontogenic sinusitis and aspergilloma can be hidden should not be overlooked.

Unilateral nasal polyposis indicates either AFRS or an inverted papilloma. Even nasal carcinomas should not be ruled 


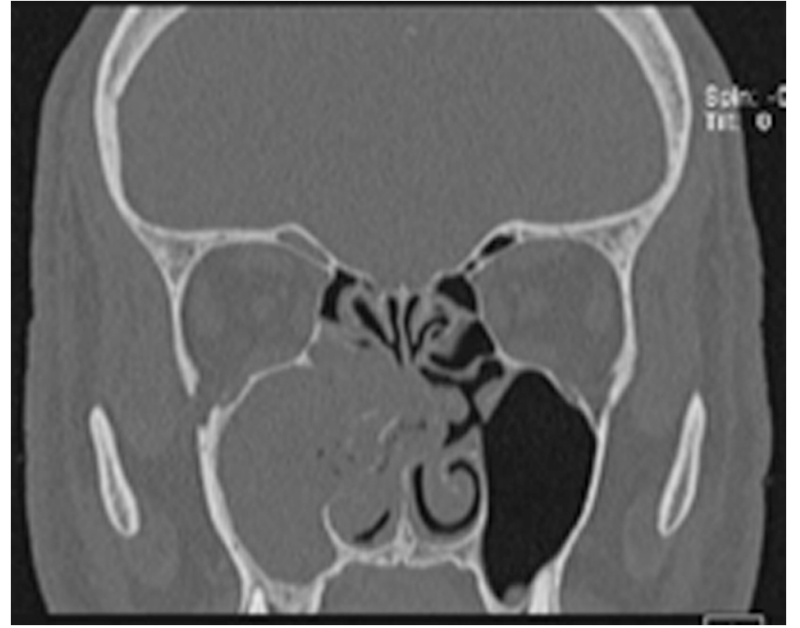

Figure 1. Coronal CT scan revealing the appearance of fungal right rhinosinusitis with full right maxillary sinus, with obstruction of the osteo-meatal complex, lysis of the medial wall, of the middle nasal cornet skeleton, partially of the bony septum and with suggestive image of aspergilloma in the right middle meatus. CT, computed tomography.

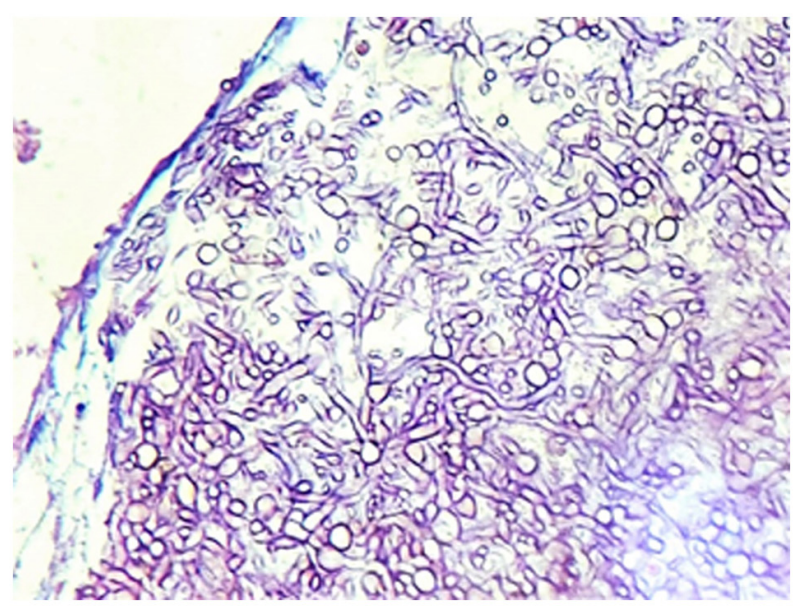

Figure 2. Hyphae and 'fruiting' (conidial) heads with non-invasive aspects. Hematoxylin and eosin staining. Magnification, $\mathrm{x} 400$.

out from the differential diagnosis. There are situations in which the initial evolution of the tumor generates the required changes in the local environment leading to the development of a fungal infection (21).

Imaging exploration is mandatory to support the diagnosis of fungal rhinosinusitis and reveals unilateral maxillo-ethmoidal rhinosinusal damage with hyperostosis of the walls of the maxillary sinus, endosinusal calcifications and obstruction of the osteomeatal complex (Fig. 6). However, the characteristic imaging lesions for an aspergilloma consists of the lysis of the bony walls of the maxillary sinus, especially of the medial wall, of the orbital floor, but also of the bony septum. A high-resolution CT scan underlines the bone involvement with erosions. Magnetic resonance imaging (MRI) should also be performed in cases where the erosion is tackling the skull base to rule out meningeal involvement. In addition, MRI is useful in delineating the level of fluid retention inside the sinus cavity secondary to tumor obstruction (22).

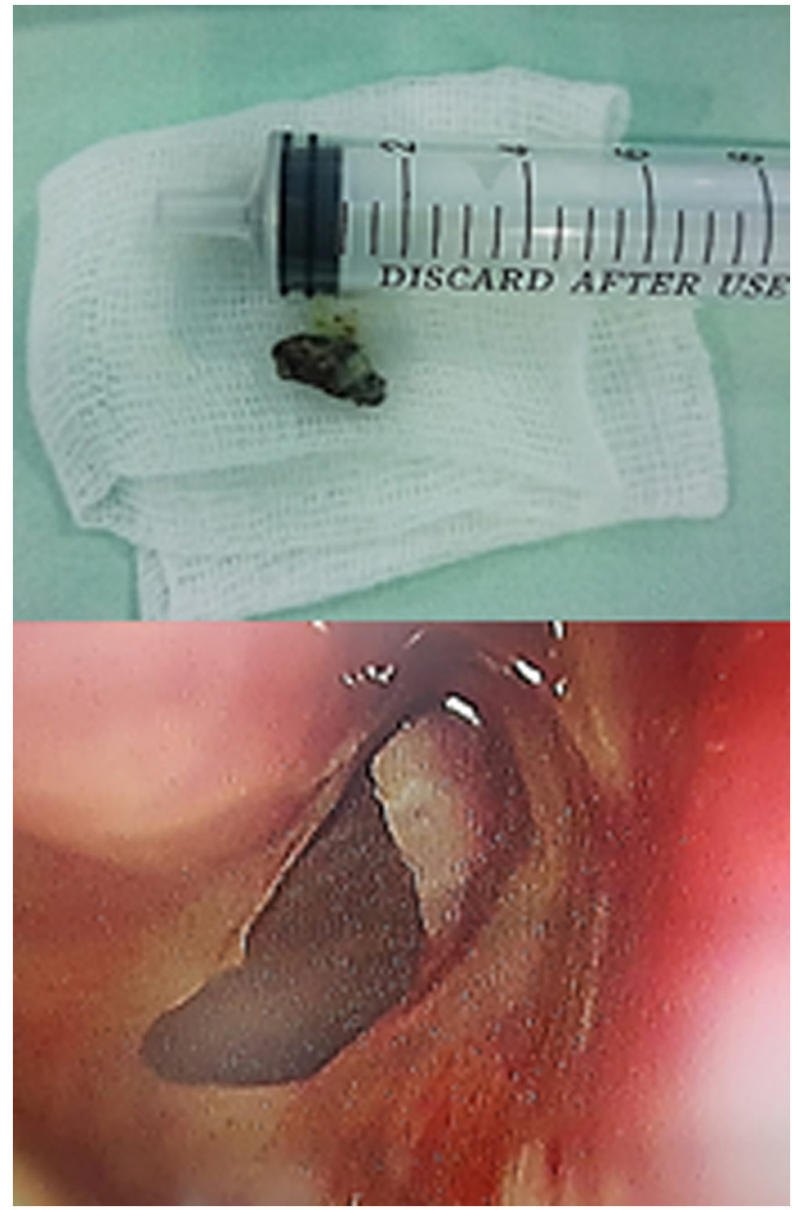

Figure 3. Classic image of the aspergilloma extracted endoscopically from the right sphenoid sinus and final surgical image with normal mucosa aspect.

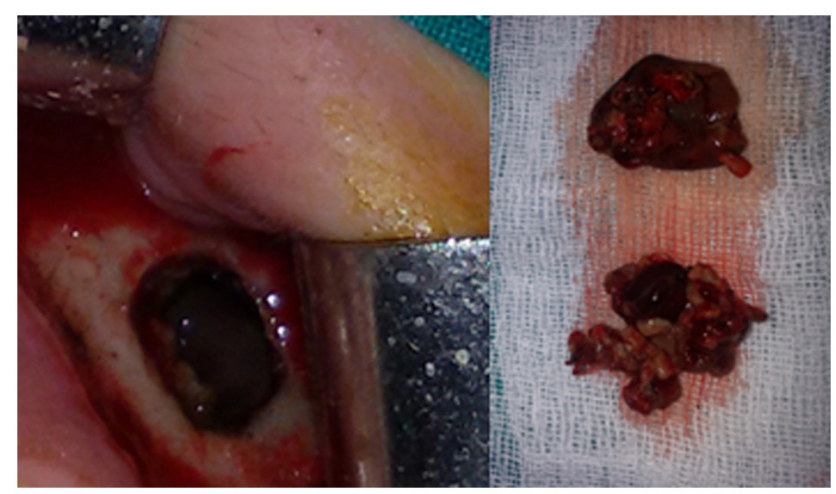

Figure 4. Surgical aspect with necrotic maxillary bone and gelatinous modified content of the mucosa in an invasive form of aspergilloma.

Performing rhinosinusal imaging should always be completed when there is unilaterally suspected clinical and imaging unilateral rhinosinusitis with orthopantomogram or cone beam computed tomography (CBCT) to highlight a dental outbreak in the sinus teeth to be treated concomitantly with surgical treatment of aspergilloma or more rarely conservatively, shortly after aspergilloma treatment to prevent a recurrence of sinus fungal injury (Fig. 7). Surgery represents the option only after receiving the clinical, endoscopic and imaging results (23). 


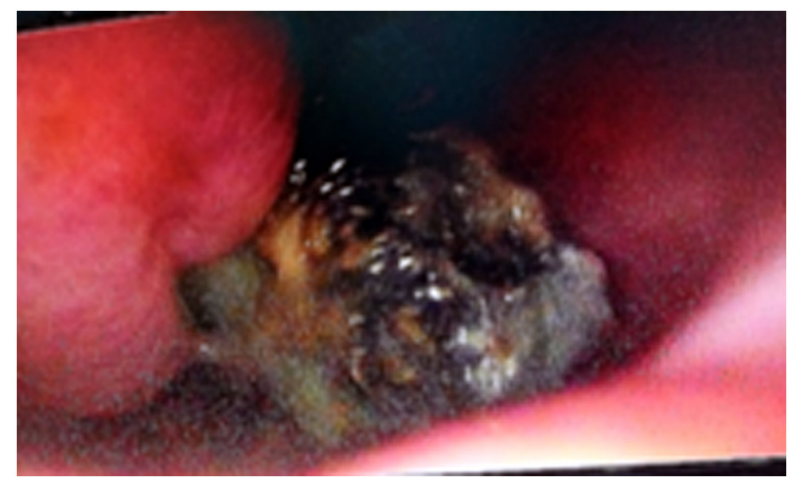

Figure 5. Endoscopic image of the aspergilloma situated in the sphenoid ostium along with puss secretion.

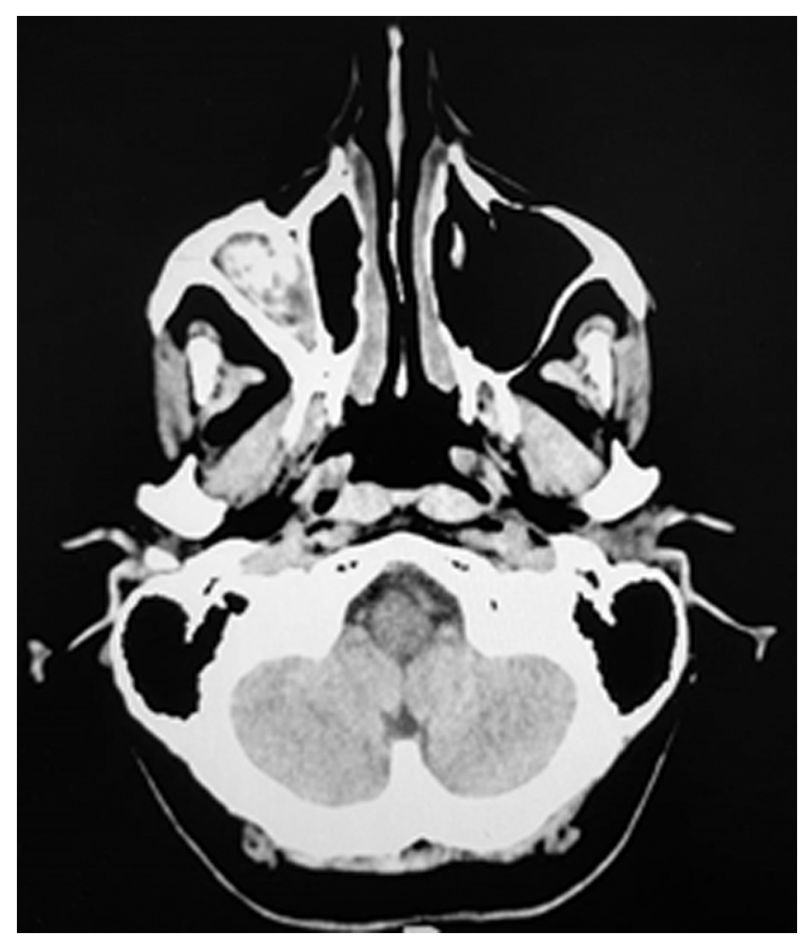

Figure 6. Aspergilloma in the right maxillary sinus with the development of a bony septum and evolution of the fungal infection in the lateral compartment.

The biological picture is not very suggestive in the immunocompetent host which presents an increase in erythrocyte sedimentation rate (ESR) and occasionally an eosinophilia associated with aspergillomas can be revealed in the context of AFRS. Numerous cases also associate with uncontrolled diabetes (24).

In the exacerbation of a chronic fungal rhinosinusitis or when the immunity of the patient becomes precarious, the clinical onset can be witnessed through a most common oculo-orbital complication with orbital cellulite associated especially with a violent nosebleed, with an intense headache, possible fever and a general impaired condition (Fig. 8). In such cases, the biological picture reveals an acute inflammatory syndrome, and the therapeutic attitude requires emergency surgical drainage and massive antibiotic therapy. On such occasions, aspergilloma is an intraoperative discovery that may require the perioperative association with a systemic

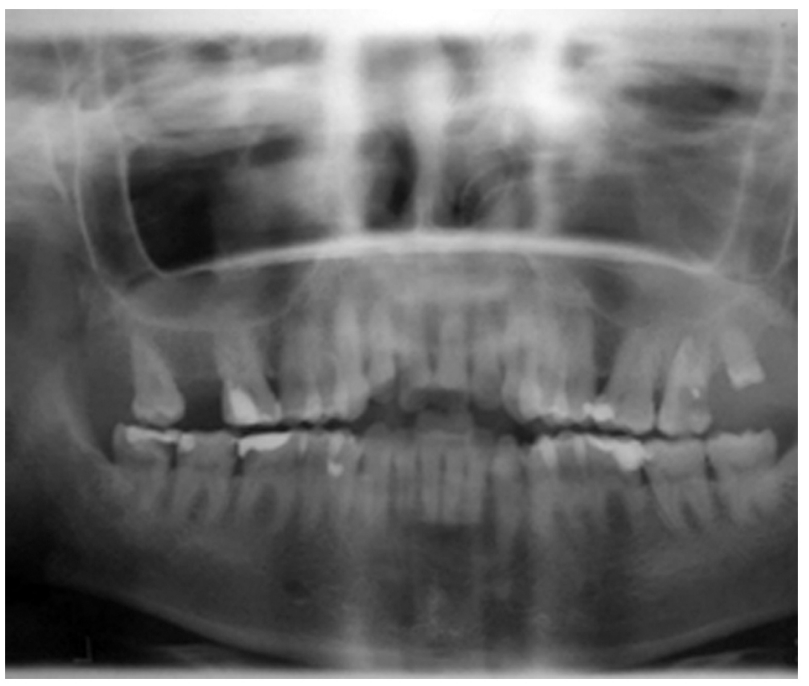

Figure 7. Dental panoramic X-ray with apical granuloma on the same side with the right sinus pathology.

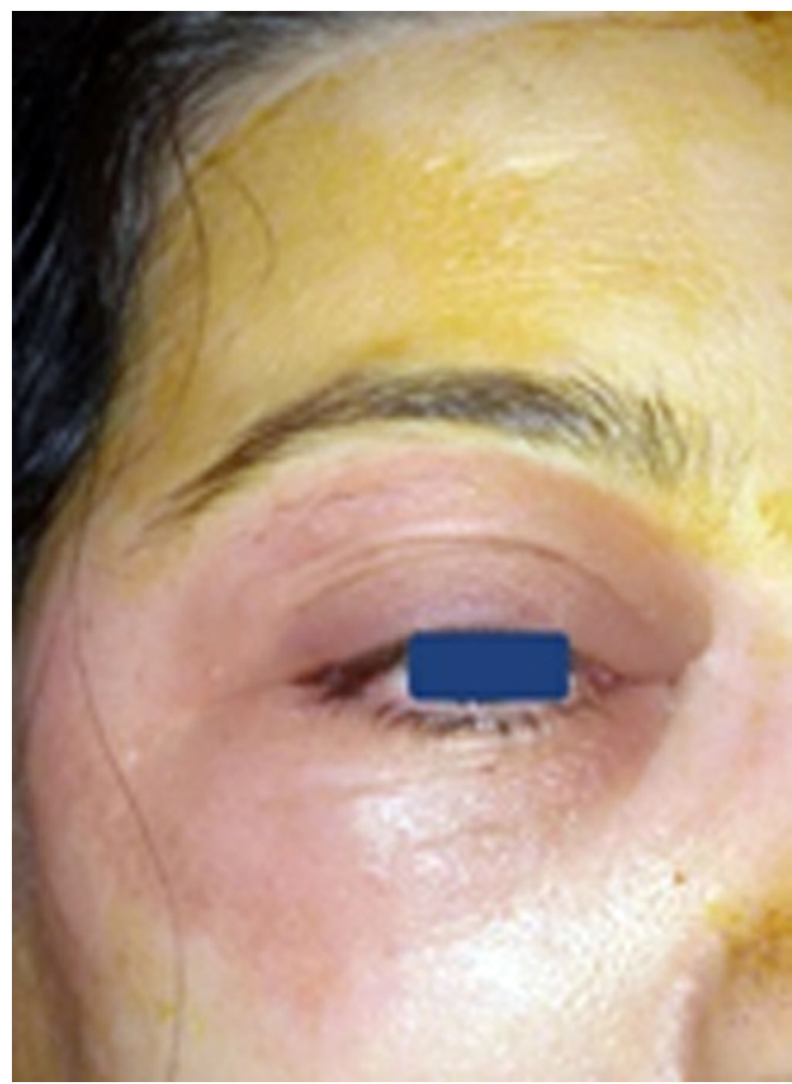

Figure 8. Clinical aspect of invasive right maxillary sinus aspergilloma in an immunocompromised host with complicated right orbit cellulitis.

antifungal. Voriconazole is one of the compounds used on a wide scale for emergency cases due to its diminished systemic toxicity (25).

\section{Principles of treatment in rhinosinusal aspergilloma}

In general, the treatment is based on surgical debridement and systemic steroids. Allergic sensitization reduction and 


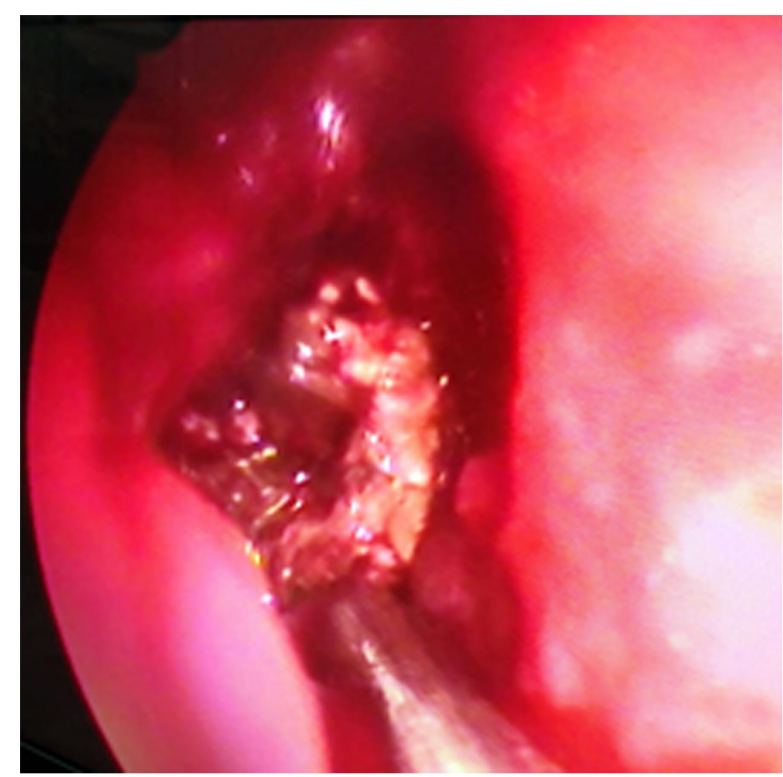

Figure 9. Endoscopic image depicting the removal of a right middle meatus aspergilloma.

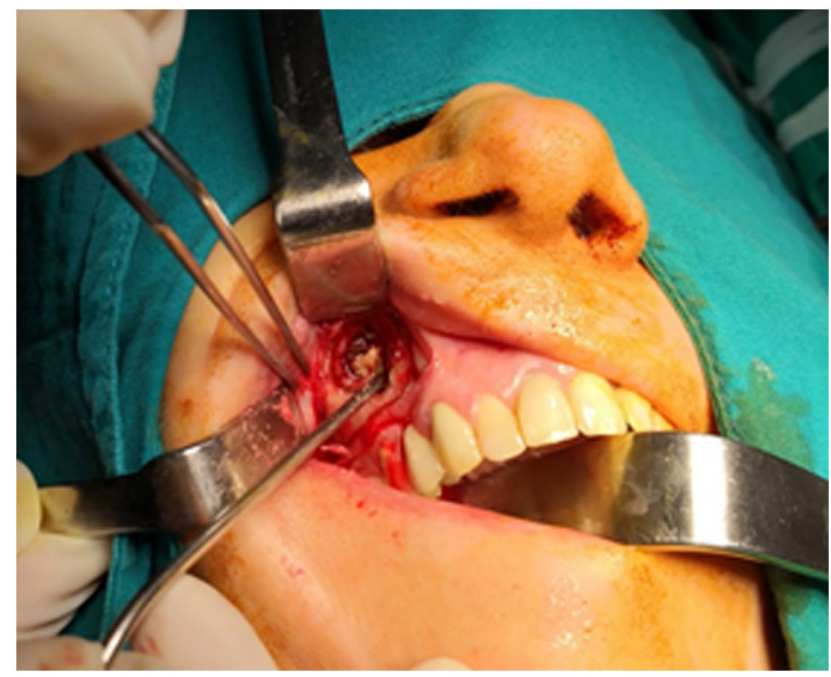

Figure 10. Surgical aspect of a giant aspergilloma occupying the entire sinus cavity.

antifungal treatment are controversial with benefits in specific cases (26).

The surgical approach should be either external or combined with endoscopy taking into consideration the site of infection, patient status, as well as the experience of the surgeon. For small aspergillomas of the middle meatus or spheno-ethmoidal recess, endoscopic extraction is possible with correct vision and radical ablation of the lesion associated, ensuring the drainage and ventilation of the involved sinus (Fig. 9). In aspergillomas of the maxillary sinus, the external approach conveys some advantages compared with Functional Endoscopic Sinus Surgery (FESS), by enabling complete removal of the fungus and drainage of the remnant sinus cavity (Fig. 10). Moreover, wide external exposure allows complete ablation of the sinus mucosa containing all aspergillus colonies and preventing recurrence (Fig. 11) (27). The

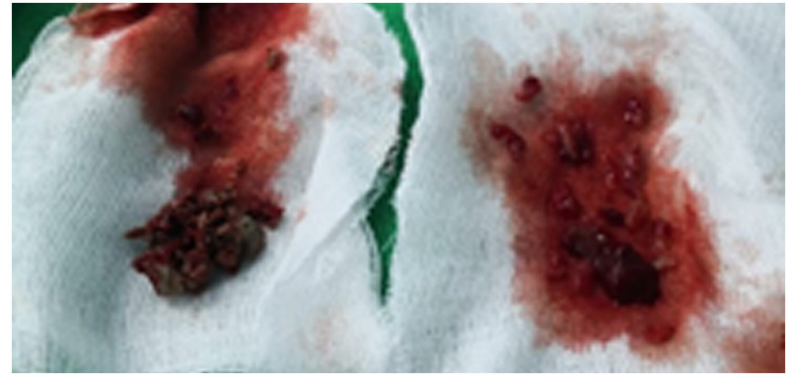

Figure 11. Surgical sample showing aspergilloma plus adjacent modified mucosa.

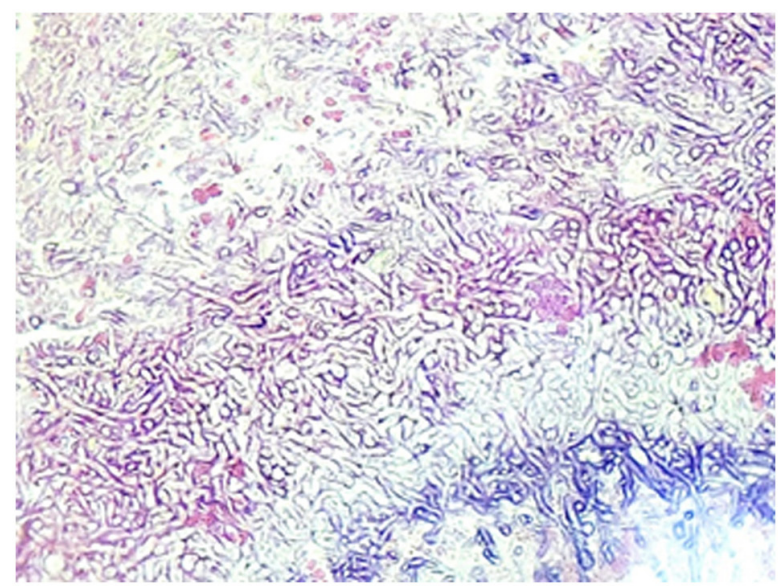

Figure 12. Fungus ball characteristic of Aspergillus sp. Hematoxylin and eosin staining. Magnification, x200.

bacteriological examination with antibiogram of the secretion collected intraoperatively from the maxillary sinus for aerobic and anaerobic flora for targeted antibiotic therapy is mandatory, as well as mycological examination. Nevertheless, histological examination is what provides certainty, by describing Aspergillus colonies in intraoperative samples. A fungus ball is the characteristic non-invasive aspect of aspergilloma, histologically characterized by colonies with hyphae and so called 'fruiting' (conidial) heads (Fig. 12). Frequently, aspergillomas in the maxillary sinus develop due to dental pathology and it is frequently necessary to extract the affected teeth and close oral fistulas. This is another situation that requires the external approach or a combined endoscopic approach (28).

Medical treatment includes antibiotics covering the spectrum of anaerobic microorganisms. The combination of $3 \mathrm{rd}$ generation cephalosporins with metronidazole is useful. This combination penetrates the blood-brain barrier and prevents endocranial and orbit extension of the infectious process. Such combinations should be administered even in cases without complications upon admission (29).

Antifungal compounds should be used in cases with compromised immune response. Given the availability in the hospital, voriconazole, caspofungin, or amphotericin B should be opted for (30). Voriconazole is most widely used due to its favorable tolerance by the patients and high efficiency improving survival with diminished systemic toxicity (31).

In cases with efficient immune response, administering oral antifungal compounds with fewer side effects should be 
considered. Compounds like itraconazole or posaconazole should be administered up to 10 days after surgery. However, these compounds are inefficient without surgical removal of the fungus ball (32).

For the endoscopic approach of the rhinosinusal aspergilloma, special equipment with wide angle nasal endoscopes is necessary, for a clear view of the sinus cavities (33). Moreover, the endoscopic approach enables reaching pathology positioned deeper in the ethmoid cells (34). This is a minimally invasive approach (35). In cases of hematologic malignancies and higher risk of bleeding, the endoscopic approach appears safer (36). The recovery after the endoscopic approach appears to be more rapid and thorough in chronic cases (37). Fortunately, this type of pathology appears to have a lower incidence in Eastern Europe and the cases should be referred to regional tertiary specialized units, with experience in the management of such cases (38).

\section{Conclusions}

Rhinosinusal aspergilloma has a slow, insidious evolution over months and even years. Our experience revealed the presence of both a dental problem and previous self-administered antibiotic regimens in almost every case. The initial symptoms are common with sinusitis of dental origin, but aspergilloma should be considered when a patient with a competent immune system does not respond to standard antibiotic treatment.

A comprehensive review of a special type of fungal rhinosinusitis caused by Aspergillus strains is presented. There are other fungal species that can cause rhinosinusitis as well. There is a lot of confusion regarding the classification of fungal rhinosinusitis. The most accepted classification includes acute invasive fungal rhinosinusitis, granulomatous invasive fungal rhinosinusitis, chronic invasive fungal rhinosinusitis, fungus ball, AFRS and saprophytic fungal infestation (39).

Aspergillus can cause invasive or non-invasive disease. The differentiation between invasive and non-invasive disease is important because the two entities have different treatment options and prognosis.

Laboratory results are in the normal range, except occasionally elevated ESR, and this should raise the question why the organism is not correctly fighting the fungus infection.

The final diagnosis of rhinosinusal aspergilloma is conducted on a pathology sample with silver staining. The bacteriology exam of the sinus secretion rarely reveals a fungal infection, but as revealed in our clinical experience, it may indicate a coinfection with other multidrug-resistant bacteria.

Early diagnosis and emergency surgical debridement, along with administering systemic antifungal compounds, represent in some cases the key to successful treatment of invasive aspergilloma. Surgical treatment must establish a wide exposure of the sinus cavity and correct drainage, regardless of the external or combined endoscopic approach. Voriconazole is one of the compounds used on a wide scale for emergency cases, due to its diminished systemic toxicity.

\section{Acknowledgements}

Professional editing, linguistic and technical assistance was performed by Irina Radu, Individual Service Provider, certified translator in Medicine and Pharmacy (certificate credentials: E0048/2014).

\section{Funding}

No funding was received.

\section{Availability of data and materials}

All data generated or analyzed during this study are included in this published article.

\section{Authors' contributions}

DV, MD and TP contributed substantially to the conception and design of the study, the acquisition, analysis, and interpretation of the data, and were involved in the drafting of the manuscript. OMP and AC contributed substantially to the acquisition, analysis and interpretation of the data and were involved in the drafting of the manuscript. RC contributed substantially to the acquisition of the data and was involved in the critical revisions of the manuscript for important intellectual content. All authors agreed to be accountable for all aspects of the work in ensuring that questions related to the accuracy or integrity of any part of the work are appropriately investigated and resolved. All the authors read and approved the final version of the manuscript.

\section{Ethics approval and consent to participate}

The study followed the international regulations in accordance with the Declaration of Helsinki.

\section{Patient consent for publication}

Patient informed consent for publication of the data/images associated with the manuscript was obtained. The authors followed the international regulations in accordance with the Declaration of Helsinki and all identifying information was removed.

\section{Competing interests}

The authors declare that they have no competing interests.

\section{References}

1. Neville BW, Damm DD, Allen CM and Bouquot JE: Fungal and protozoal diseases. In: Oral and Maxillofacial Pathology. 2nd edition. Saunders, Philadelphia PA, pp189-211, 2005.

2. Lin SJ, Schranz J and Teutsch SM: Aspergillosis case-fatality rate Systematic review of the literature. Clin Infect Dis 32: 358-366, 2001.

3. Nikolaizik WH, Weichel $\mathrm{M}$, Blaser $\mathrm{K}$ and Crameri R: Intracutaneous tests with recombinant allergens in cystic fibrosis patients with allergic bronchopulmonary aspergillosis and aspergillus allergy. Am J Respir Crit Care Med 165: 916-921, 2002.

4. Hartl D, Latzin P, Zissel G, Krane M, Krauss-Etschmann S and Griese M: Chemokines indicate allergic bronchopulmonary aspergillosis in patients with cystic fibrosis. Am J Respir Crit Care Med 173: 1370-1376, 2006.

5. Kraemer R, Deloséa N, Ballinari P, Gallati S and Crameri R: Effect of allergic bronchopulmonary aspergillosis on lung function in children with cystic fibrosis. Am J Respir Crit Care Med 174: 1211-1220, 2006. 
6. Frisvad JC, Rank C, Nielsen KF and Larsen TO: Metabolomics of aspergillus fumigatus. Med Mycol 47 (Suppl 1): S53-S71, 2009

7. Guarner J and Brandt ME: Histopathologic diagnosis of fungal infections in the 21st century. Clin Microbiol Rev 24: 247-280, 2011.

8. Arndt S, Aschendorff A, Echternach M, Daemmrich TD and Maier W: Rhino-orbital-cerebral mucormycosis and aspergillosis: Differential diagnosis and treatment. Eur Arch Otorhinolaryngol 266: 71-76, 2009.

9. Walsh TJ, Anaissie EJ, Denning DW, Herbrecht R, Kontoyiannis DP, Marr KA, Morrison VA, Segal BH, Steinbach WJ, Stevens DA, et al: Treatment of aspergillosis: Clinical practice guidelines of the infectious diseases society of america. Clin Infect Dis 46: 327-360, 2008

10. Tamgadge AP, Mengi R, Tamgadge S and Bhalerao SS: Chronic invasive aspergillosis of paranasal sinuses: A case report with review of literature. J Oral Maxillofac Pathol 16: 460-464, 2012

11. Sharma OP and Chwogule R: Many faces of pulmonary aspergillosis. Eur Respir J 12: 705-715, 1998.

12. Warder FR, Chikes PG and Hudson WR: Aspergillosis of the paranasal sinusis. Arch Otolaryngol 101: 683-685, 1975.

13. Sapp JP, Eversole LR and Wysocki GP: Oral infections. In Contemporary Oral and Maxillofacial Pathology. 2nd edition. Mosby, St. Louis, MO, pp207-251, 2004.

14. Dayananda BC, Vandana R, Rekha K and Kumar GS Aspergillosis of the maxillary antrum: A case report. J Oral Maxillofac Pathol 1: 26-29, 2002.

15. Chambers MS, Lyzak WA, Martin JW, Lyzak JS and Toth BB Oral complications associated with aspergillosis in patients with a hematologic malignancy. Presentation and treatment. Oral Surg Oral Med Oral Pathol Oral Radiol Endod 79: 559-563, 1995.

16. Veress B, Malik OA, el-Tayeb AA, el-Daoud S, Mahgoub ES and el-Hassan AM: Further observations on the primary paranasal aspergillus granuloma in the Sudan: A morphological study of 46 cases. Am J Trop Med Hyg 22: 765-772, 1973.

17. Samaranayake LP, Keung Leung W and Jin L: Oral mucosal fungal infections. Periodontol 2000 49: 39-59; 2009.

18. Jeican II, Barbu Tudoran L, Florea A, Flonta M, Trombitas V, Apostol A, Dumitru M, Aluas M, Junie LM and Albu S: Chronic Rhinosinusitis: MALDI-TOF'mass spectrometry microbiological diagnosis and electron microscopy analysis; Experience of the 2nd otorhinolaryngology clinic of Cluj-Napoca, Romania. J Clin Med 9: 3973, 2020.

19. Denning DW: Invasive aspergillosis. Clin Infect Dis 26: 781-803, 1998.

20. Regezi JA, Sciubba JJ and Jordan RC: Ulcerative conditions. In: Oral pathology-Clinical Pathologic Correlations. 4th edition. Saunders, St. Louis, MO, pp23-74, 2003.

21. Kwon J, Park KH, Park SI and Jin SY: Aspergillosis of the paranasal sinuses-diagnostic significance of the computed tomography. Yonsei Med J 30: 294-297, 1989.

22. Ciobanu IC, Motoc A, Jianu AM, Cergan R, Banu MA and Rusu MC: The maxillary recess of the sphenoid sinus. Rom J Morphol Embryol 50: 487-489, 2009.

23. DelGaudio JM, Swain RE, Kingdom TT, Muller S and Hudgins PA: Computed Tomographic findings in patients with invasive fungal sinusitis. Arch Otolaryngol Head Neck Surg 129 236-240, 2003

24. Rusu E, Jinga M, Rusu F, Ciurtin C, Enache G, Dragomir A, Cristescu V, Stoica V, Costache A, Cheta D and Radulian G: Statin therapy in patients with diabetes and hepatitis C. Farmacia 61: $1204-1215,2013$
25. Pasqualotto AC, Xavier MO, Andreolla HF and Linden R: Voriconazole therapeutic drug monitoring: Focus on safety. Expert Opin Drug Saf 9: 125-137, 2010.

26. Berghi NO, Dumitru M, Vrinceanu D, Ciuluvica RC, SimioniucPetrescu A, Caragheorgheopol R, Tucureanu C, Cornateanu RS and Giurcaneanu C: Relationship between chemokines and T lymphocytes in the context of respiratory allergies (Review). Exp Ther Med 20: 2352-2360, 2020.

27. Stammberger H, Jakse R and Beaufort F: Aspergillosis of the paranasal sinuses; X-ray diagnosis, histopathology, and clinical aspects. Ann Otol Rhinol Laryngol 93: 251-256, 1984.

28. Rowe-Jones JM and Moore-Gillon V: Destructive noninvasive paranasal sinus aspergillosis: Component of a spectrum of disease. J Otolaryngol 23: 92-96, 1994.

29. Myoken Y, Sugata T, Fujita Y, Fujihara M, Iwato K, Murayama SY and Mikami Y: Early diagnosis and successful management of atypical invasive aspergillus sinusitis in a hematopoietic cell transplant patient: A case report. J Oral Maxillofac Surg 64: 860-863, 2006

30. Sungkanuparph S, Sathapatayavongs B, Kunachak S, Luxameechanporn T and Cheewaruangroj W: Treatment of invasive fungal sinusitis with liposomal amphotericin B: A report of four cases. J Med Assoc Thai 84: 593-601, 2001.

31. Peral-Cagigal B, Redondo-González LM and VerrierHernández A: Invasive maxillary sinus aspergillosis: A case report successfully treated with voriconazole and surgical debridement. J Clin Exp Dent 6: e448-e451, 2014.

32. Herbrecht R, Denning DW, Patterson TF, Bennett JE, Greene RE, Oestmann JW, Kern WV, Marr KA, Ribaud P, Lortholary O, et al: Voriconazole versus amphotericin B for primary therapy of invasive aspergillosis. N Engl J Med 347: 408-415, 2002.

33. Lee JH, Oh DH and Lee DH: A single small-sized fungus ball in the maxillary sinus: An endoscopic view. Ear Nose Throat J 99: $165-166,2020$.

34. Lee JH: Endoscopic view of fungus ball in the most posterior ethmoid cell via the supreme meatus. Ear Nose Throat J 98: 319-320, 2019.

35. Mitroi M, Albulescu D, Capitanescu A, Docea AO, Musat G, Mitroi G, Zlatian O, Tsatsakis A, Tzanakakis G, Spandidos DA and Calina D: Differences in the distribution of CD20, CD3, CD34 and CD45RO in nasal mucosa and polyps from patients with chronic rhinosinusitis. Mol Med Rep 19: 2792-2800, 2019.

36. Gletsou E, Ioannou M, Liakopoulos V, Tsiambas E, Ragos V and Stefanidis I: Aspergillosis in immunocompromised patients with haematological malignancies. J Buon 23: 7-10, 2018.

37. Jeican II, Trombitas V, Crivii C, Dumitru M, Aluas M, Dogaru G, Gheban D, Junie LM and Albu S: Rehabilitation of patients with chronic rhinosinusitis after functional endoscopic sinus surgery. Balneo and PRM Research Journal 12: 65-72, 2021.

38. Leszczyńska J, Stryjewska-Makuch G, Lisowska G, Kolebacz B and Michalak-Kolarz M: Fungal sinusitis among patients with chronic rhinosinusitis who underwent endoscopic sinus surgery. Otolaryngol Pol 72: 35-41, 2018

39. Deutsch PG, Whittaker J and Prasad S: Invasive and non-invasive fungal Rhinosinusitis-A review and update of the evidence. Medicina (Kaunas) 55: 319, 2019.

his work is licensed under a Creative Commons Attribution-NonCommercial-NoDerivatives 4.0 International (CC BY-NC-ND 4.0) License. 